\title{
Lamellar keratoplasty using position- guided surgical needle and M-mode optical coherence tomography
}

Sungwon Shin

Jung Kweon Bae

Yujin Ahn

Hyeongeun Kim

Geonho Choi

Young-Sik Yoo

Choun-Ki Joo

Sucbei Moon

Woonggyu Jung 


\title{
Lamellar keratoplasty using position-guided surgical needle and M-mode optical coherence tomography
}

\author{
Sungwon Shin, ${ }^{\text {a, } \dagger}$ Jung Kweon Bae,,${ }^{a} \dagger$ Yujin Ahn, ${ }^{a}$ Hyeongeun Kim, ${ }^{a}$ Geonho Choi, ${ }^{a}$ Young-Sik Yoo, ${ }^{b}$ \\ Choun-Ki Joo, ${ }^{b}$ Sucbei Moon, ${ }^{c, *}$ and Woonggyu Jung ${ }^{a, *}$ \\ aUlsan National Institute of Science and Technology, Department of Biomedical Engineering, Ulsan, Republic of Korea \\ ${ }^{b}$ Catholic University of Korea, College of Medicine, Department of Ophthalmology and Visual Science, Seoul, Republic of Korea \\ 'Kookmin University, Department of Physics, Seoul, Republic of Korea
}

\begin{abstract}
Deep anterior lamellar keratoplasty (DALK) is an emerging surgical technique for the restoration of corneal clarity and vision acuity. The big-bubble technique in DALK surgery is the most essential procedure that includes the air injection through a thin syringe needle to separate the dysfunctional region of the cornea. Even though DALK is a well-known transplant method, it is still challenged to manipulate the needle inside the cornea under the surgical microscope, which varies its surgical yield. Here, we introduce the DALK protocol based on the position-guided needle and M-mode optical coherence tomography (OCT). Depth-resolved 26-gage needle was specially designed, fabricated by the stepwise transitional core fiber, and integrated with the swept source OCT system. Since our device is feasible to provide both the position information inside the cornea as well as air injection, it enables the accurate management of bubble formation during DALK. Our results show that real-time feedback of needle end position was intuitionally visualized and fast enough to adjust the location of the needle. Through our research, we realized that position-guided needle combined with M-mode OCT is a very efficient and promising surgical tool, which also to enhance the accuracy and stability of DALK. $\odot$ The Authors. Published by SPIE under a Creative Commons Attribution 3.0 Unported License. Distribution or reproduction of this work in whole or in part requires full attribution of the original publication, including its DOI. [DOI: 10.1117/1.JBO.22.12.125005]
\end{abstract}

Keywords: medical imaging probe; surgical guidance; ophthalmology; deep anterior lamellar keratoplasty; M-mode optical coherence tomography.

Paper 170574R received Aug. 30, 2017; accepted for publication Nov. 20, 2017; published online Dec. 13, 2017

\section{Introduction}

Lamellar keratoplasty (LK) is a transplant procedure used to treat corneal diseases or injuries with a lower rejection and higher integrity of the cornea after transplantation. ${ }^{1-6}$ It enables the partial replacement of a recipient's corneal tissue with that of a donor. In the case of deep anterior lamellar keratoplasty (DALK), dysfunctional stroma of cornea is replaced after being clearly separated from the Descemet's membrane (DM). The success rate for DALK surgery has been critically influenced by the extent of the uniformity and clearness of remaining lower layers. Since a human cornea is thin, typically $<1 \mathrm{~mm}$, the separation process needs a specialized surgical technique.

Anwar's big-bubble technique has been proven to be the most successful for this purpose. ${ }^{7-10}$ In the big-bubble technique, air is injected through a thin syringe needle that is inserted inside the patient's cornea to make the uniform corneal segments by inflating the air bubble in the cornea. Here, inserting the needle in the cornea is a potentially deadly part of the procedure. The needle may produce a fatal perforation if inserted too deeply, or no big-bubble may be formed if the

\footnotetext{
*Address all correspondence to: Sucbei Moon, E-mail: moons @ kookmin.ac.kr Woonggyu Jung, E-mail: wgjung@unist.ac.kr

†These authors contributed equally to this work.
}

air is injected at a shallow position. So far, the needle insertion has been performed with no direct information given about the depth of the needle's tip but just with help of en-face visual guidance from the surgical microscope. As a consequence of the required precision of the needlework in DALK, the surgeon could risk perforating the corneal tissue. ${ }^{11,12}$ Moreover, it is difficult to predict the visual outcome because of the presence of uneven residual stromal thickness. ${ }^{13,14}$

To assist the sophisticated big-bubble formation, the use of intraoperative optical coherence tomography (iOCT) $)^{15-19}$ or iOCT with a surgical microscope $\mathrm{e}^{20-23}$ imaging system has been considered for the DALK procedures. Monitoring the needle's manipulations in the cornea can safely handle a sharp needle and better determine the injection point with knowledge of the depth. However, its practical use is still hindered by many factors. Tracking the needle tip in the OCT systems is a cumbersome task for both a human operator and a fully computerized system. Note that real-time three-dimensional (3-D) data covering the entire surgical area are difficult to be presented in a comprehensible form without heavy processing. Moreover, opaqueness of the metallic needle for the DALK surgery makes underlying tissue invisible in the conventional OCT imaging schemes. $^{24}$ Therefore, the acquired OCT information is still limited in contents, difficult to comprehend, and vulnerable to the environmental factors in the real-world operation room. We believe that there is still room to further improve the entire surgical procedure by OCT visualization means. ${ }^{25,26}$ 
In this article, we introduce a position-guided needle combined with M-mode OCT for the DALK procedures. We developed a needle that is internally equipped with an ultrathin fiber-based OCT probing optics. Our needle utilized its OCT principle that acquires the back-reflected OCT light through the needle's bevel outlet. In addition, free-hand manual scanning was employed in axial direction to display the dynamically varying location of the needle tip over the time (M-mode). Whereas the OCT needles developed in the previous studies were mainly for diagnostic imaging, ${ }^{27-31}$ our needle was used for fluid injection just like the ordinary syringe needles as well as position sensing. The performance of our system was initially evaluated through a cornea mimicking phantom, and its applicability in real ophthalmic surgery was identified in the animal model ex vivo.

\section{Materials and Methods}

\subsection{Position-Guided Surgical Needle and M-Mode Optical Coherence Tomography}

We developed a position-guided surgical needle, which equips an embedded OCT that can acquire the positional contexts of the underlying tissue. For a compact and minimalistic structure, our surgical needle employed a stepwise transitional core (STC) fiber [Fig. 1(a)], which produces an output beam without using a lens element. ${ }^{32}$ It was composed of three different optical fibers with an outer diameter of $125 \mu \mathrm{m}$, spliced in series to the standard single-mode fiber of light delivery. The large core fiber with its diameter of $29 \mu \mathrm{m}$ at the distal end directly determined the characteristics of the output beam while keeping the effective single-mode guidance through the STC structure.
To bend the beam perpendicularly to the fiber axis, a 43 degtilted reflector plane was made by polishing the end of the fiber structure. This structure is advantageous in its compactness, ease of fabrication, and operational reliability, though with a moderate sacrifice of the imaging range. The STC fiber supported an effective imaging range of $0.9 \mathrm{~mm}$ with beam diameter of $\sim 25 \mu \mathrm{m}$ in corneal tissue $(n=1.35),{ }^{32}$ so was sufficient for our application to corneal imaging. The fabricated fragile fiber structure was reinforced by a metal jacket with an outer diameter of $200 \mu \mathrm{m}$. This fabricated fiber was finally integrated into a 26-G syringe needle as shown in Fig. 1(b). The inner and outer diameters of the position-guided needle were 260 and $460 \mu \mathrm{m}$, respectively. The fluid channel was formed between the metal-jacketed fiber and the inner side of the syringe needle.

In this study, to acquire the depth information from the needle, we utilized the home-built swept source OCT (SS-OCT) imaging system as shown in Fig. 1(c). The swept source laser (Axsun Technologies Inc.) with 1310-nm center wavelength and 105-nm bandwidth was incorporated into the SSOCT system, providing average $20 \mathrm{~mW}$ of output power and an axial resolution of $\sim 7.6 \mu \mathrm{m}$. A 90:10 coupler splits the light emitted from the swept source laser into the mirror in the reference arm and sample arm. The position-guided needle was attached to the sample arm in our system to achieve optical signals from the inside of the tissue. The power from the sample arm was measured $\sim 10 \mathrm{~mW}$. Reflections from each arm were combined and generated the spectral interference signals at the 50:50 coupler. A balanced photo detector (PDB450C, Thorlabs Inc.) and digitizer (ATS9350, Alazar Technologies Inc., Canada) were utilized to detect the interference signals. A fast Fourier transform was computed to achieve the position profile and image was updated based on time order with $\sim 0.01 \mathrm{~s}$ of update
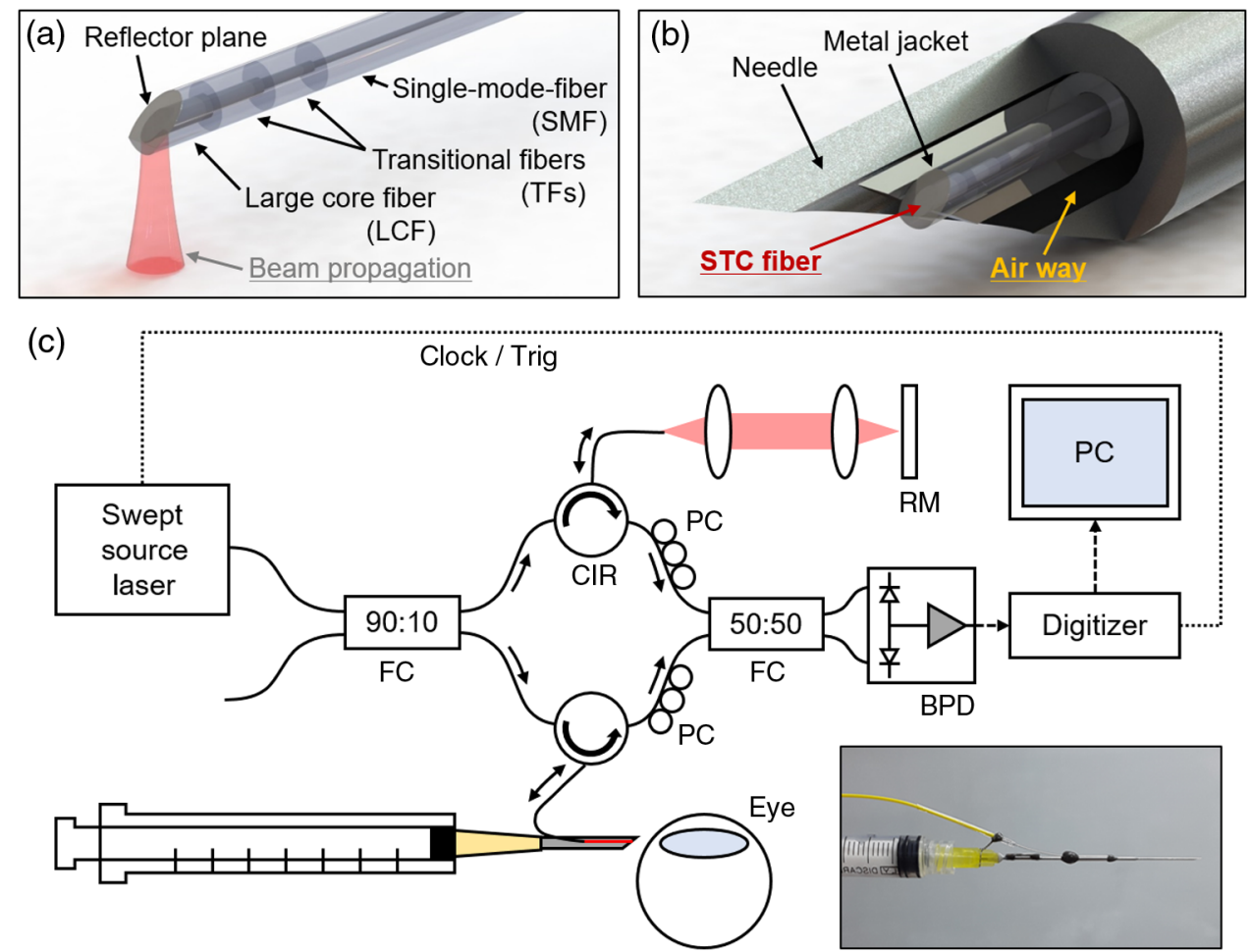

Fig. 1 Schematics of (a) STC fiber, (b) STC fiber integrated position-guided needle, and (c) customized optical coherence tomography system assembled with position-guided needle. FC, fiber coupler; CIR, circulator; PC, polarization controller; RM, reference mirror; and BPD, balanced photodetector. 
speed (M-mode). Our customized SS-OCT system facilitates 50-kHz A-line acquisition.

\subsection{Corneal-Mimicking Phantom Preparation}

To verify the functionalities of the developed position-guided surgical needle, we prepared the corneal-mimicking phantom, which has two layers, representing the cornea and the anterior chamber, respectively. The top layer was prepared by blending an agarose powder and distilled water, and a scatterer was added to the mixture to increase the optical scattering while imitating the stroma texture. The bottom layer had a low concentration of mixture compared with the top layer to realize the transparency similar to that of the anterior chamber. Solidifying the subsequently poured mixtures of differently concentrated agarose develops the corneal-mimicking phantom. The top layer of corneal-mimicking phantom was fabricated with 1-mm thickness and then utilized to evaluate the functions of the developed position-guided needle.

\subsection{Ex Vivo Animal Study Preparation}

The rabbit eyes were enucleated from two New Zealand rabbits weighing 2.0 to $2.5 \mathrm{~kg}$ that were euthanized in accordance with institutional guidelines and approved Institutional Animal Care and Use Committee at the College of Medicine, the Catholic University of Korea. The rabbit eye was held in an anterior chamber manufactured by a 3-D printer for an optimal surgical procedure. The anterior chamber consisted of a plastic cradle with two nozzles, which controlled pressure using water and air to fasten the eye tightly. After setting the eye in the cradle, a position-guided needle was inserted into the cornea, which measured the remaining thickness of the cornea in real-time when the needle end was moving toward a border between the cornea and the anterior chamber. When the needle end

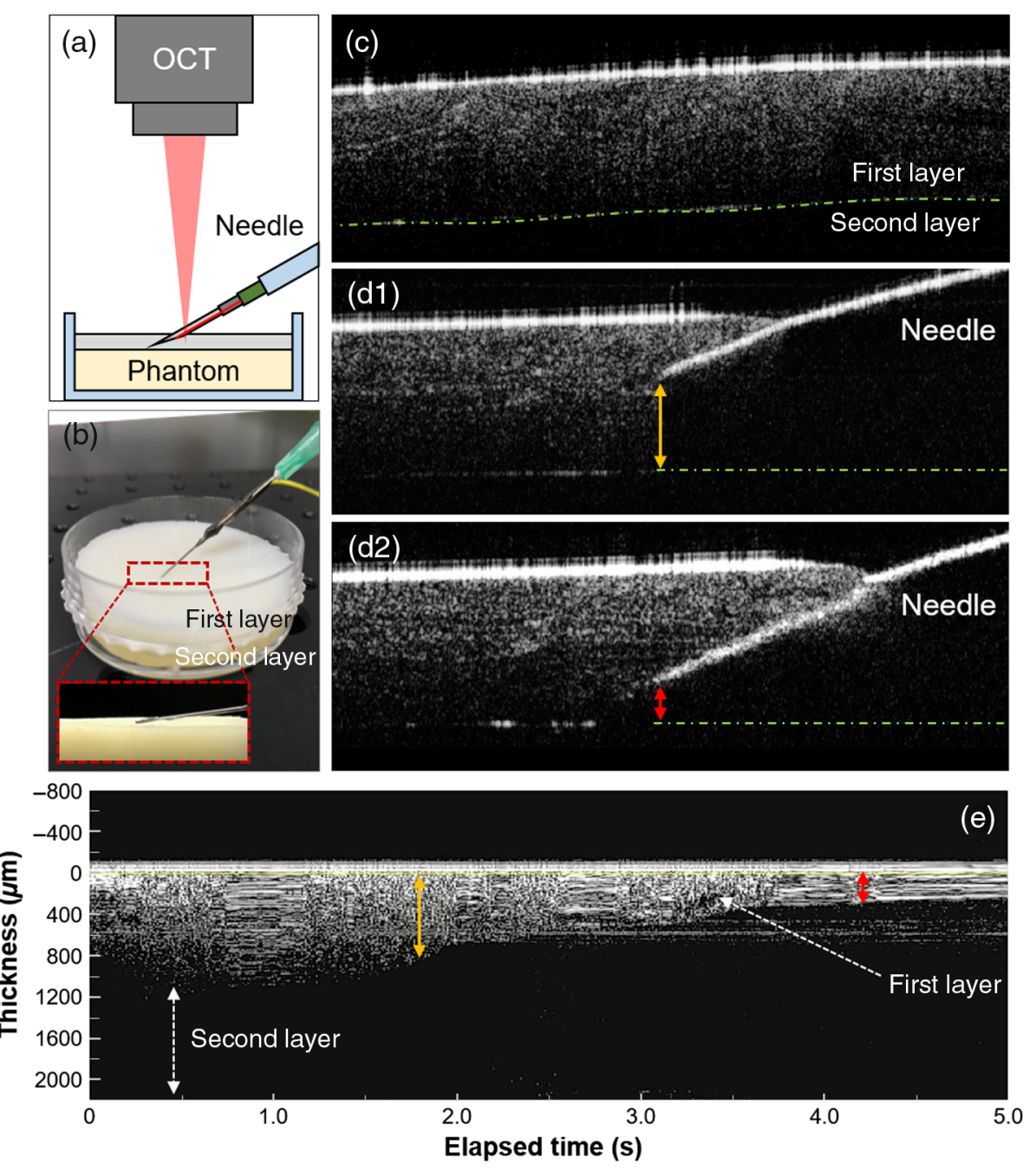

Fig. 2 Phantom study evaluating the feasibility of the position-guided needle combined with M-mode OCT. (a) Schematic diagram of phantom study. (b) Corneal-mimicking phantom developed with double layers of different concentrated agarose. (c), (d1), and (d2) regular OCT image acquired while needle punched through the surface of agarose gel and passed by the middle of first layer at $1.68 \mathrm{~s}$, and came closer to second layer at $4.14 \mathrm{~s}$, respectively. (e) M-mode imaging from position-guided needle in realtime (Video 1). Red and yellow arrows denote the residual thickness between the needle distal end and the boundary (assuming the DM and endothelium) within the phantom, respectively (Video 1, MPEG, 3.42 MB [URL: http://dx.doi.org/10.1117/1.JBO.22.12.125005.1]). 
reached the target location inside the cornea, air was blown through the needle to separate two layers.

\section{Results}

\subsection{Evaluation of Phantom Study}

To identify the performance of the surgical needle as a position sensor, the corneal-mimicking phantom was utilized, as shown in Figs. 2(a) and 2(b). For this experiment, an OCT image was taken to monitor the needle insertion in the phantom and calibrate the position information acquired from the needle. Figure 2(c) shows an OCT image of the phantom without a needle and clearly delineates the two solidified agarose layers with different concentrations and scatters. When the surgical needle is inserted into the phantom, an OCT image and position information from the needle were simultaneously acquired, as presented in Figs. 2(d) and 2(e). The A-line profile indicating the position information of the needle is directly converted and visualized as an M-mode OCT image. Video 1 presents demonstration of M-mode OCT when the position-guided needle is applied to the cornea phantom and approaches the desired location. The M-mode OCT image, which was updated every $10 \mathrm{~ms}$, denotes the time sequential signals along the depth from the end of the STC fiber. As the needle penetrated into the phantom, the thickness of the M-mode OCT image gets thinner while showing the residual thickness of the top layer. In this experiment, we confirmed that a position-guided needle enables us to clearly distinguish the two layers, and offers position location with high accuracy. The sensing speed was also fast enough to apply to real surgical settings while guiding the placement of a needle.

\subsection{Evaluation of Animal Study}

The M-mode OCT needle was applied to the DALK procedure with an ex vivo rabbit animal model with manual scanning. In this experiment, a position-guided needle with the bevel facing downward was carefully penetrated tangentially into a paracentral rabbit cornea. The entire DALK procedure was recorded by video and M-mode OCT as shown in Video 2. Figure 3 shows how the surgical needle is inserted and guided to approach near the DM. A time-sequential A-line profile acquired from the distal end of the needle was continuously monitored while updating the current location. The real-time feedback from the inserted needle was visualized on the display to help the clinician perceive the position of the needle. The whole cornea thickness was visualized at the epithelial layer [Fig. 3(a)] and became thinner when the needle approached the paracentral cornea [Fig. 3(b)]. The green line shown in the Video 2 indicates the current residual thickness between the DM and the needle end, which provide fast and intuitive information to the clinician.

We further evaluated the feasibility of bubble formation induced by our needle using SS-OCT imaging system. $512 \times$ $500 \times 500$ voxel of OCT images were captured immediately after the needle was placed at $100 \mu \mathrm{m}$ above the DM layer and after performing the air injection at the same position. Figures 4(a) and 4(b) show the DALK process including needle insertion and the various bubble formations. As shown in Figs. 4(c) and 4(d), the 3-D OCT image presents the whole structure of the cornea with the needle. In DALK surgery, a clinician usually lays the needle parallel to the corneal surface to avoid the risk of perforation under the DM layer. This method may also help to easily separate the corneal layer with less damage in stromal layer when the big-bubble technique is used. 2-D and 3-D OCT images clearly identify the needle location inside
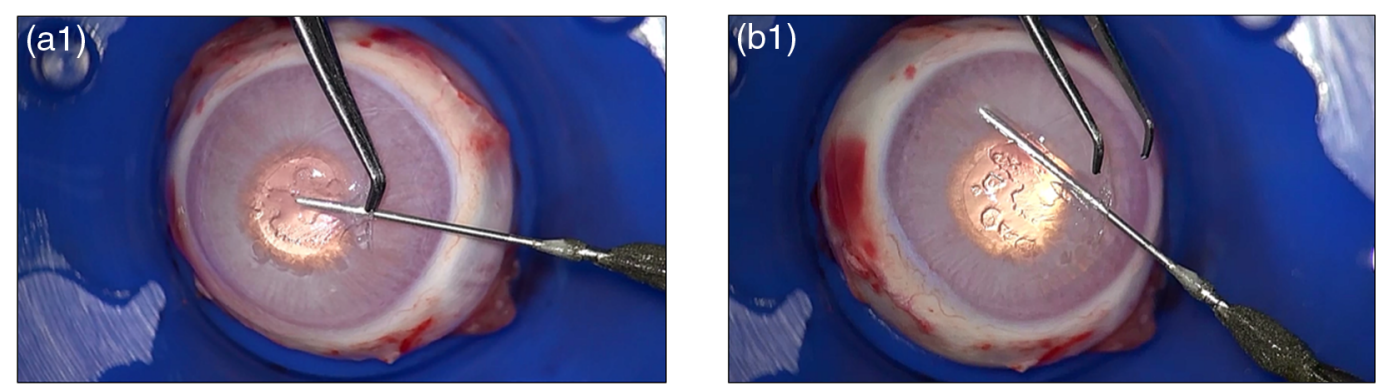

(a2)

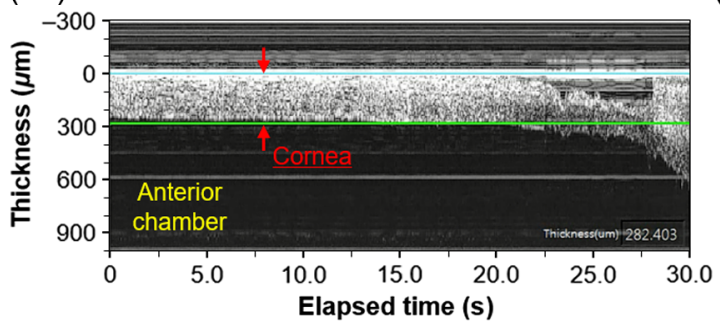

(b2)

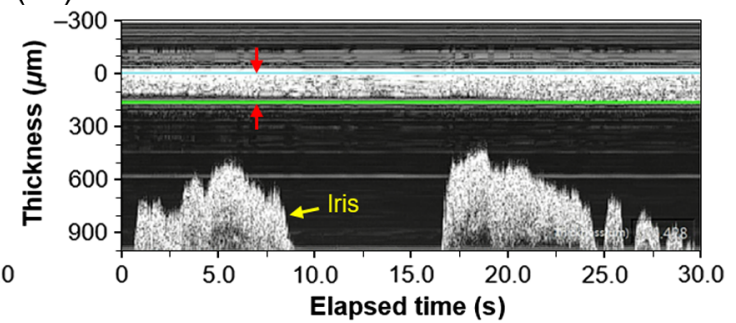

Fig. 3 Animal study demonstrating the position-guided surgical needle and M-mode OCT (Video 2). (a1) Animal surgery using position-guided needle when the tip of needle was inserted into the surface of cornea. (a2) The corresponding M-mode OCT image from the needle when its tip was maintaining the corneal layer with residual thickness of around $300 \mu \mathrm{m}$. (b1) Next medical procedure when the clinician was aided with the needle to quantitatively find out the desired position of the cornea. (b2) The corresponding M-mode OCT image was captured when the needle approached toward lowermost of the enucleated rabbit eyeball and detected the rest of the cornea thickness within $100 \mu \mathrm{m}$ (Video 2, MPEG, 6.32 MB [URL: http://dx.doi.org/10.1117/1.JBO.22.12.125005.2]). 

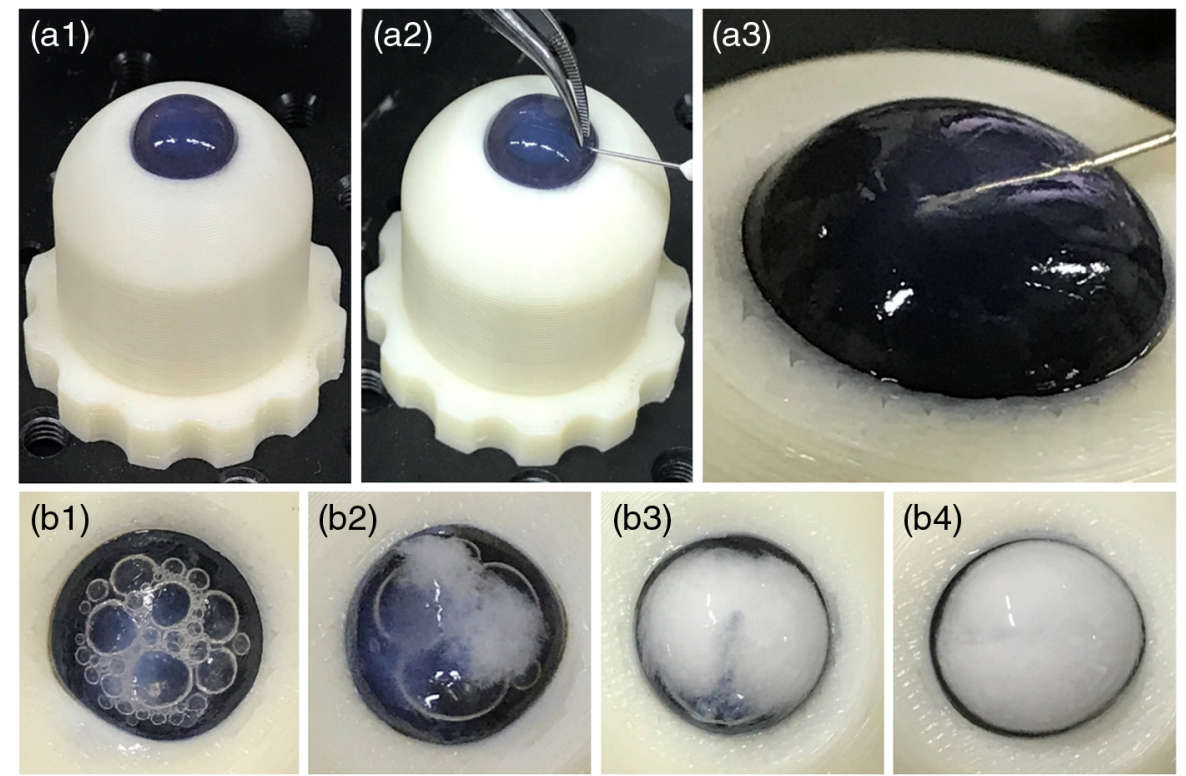

(b3)
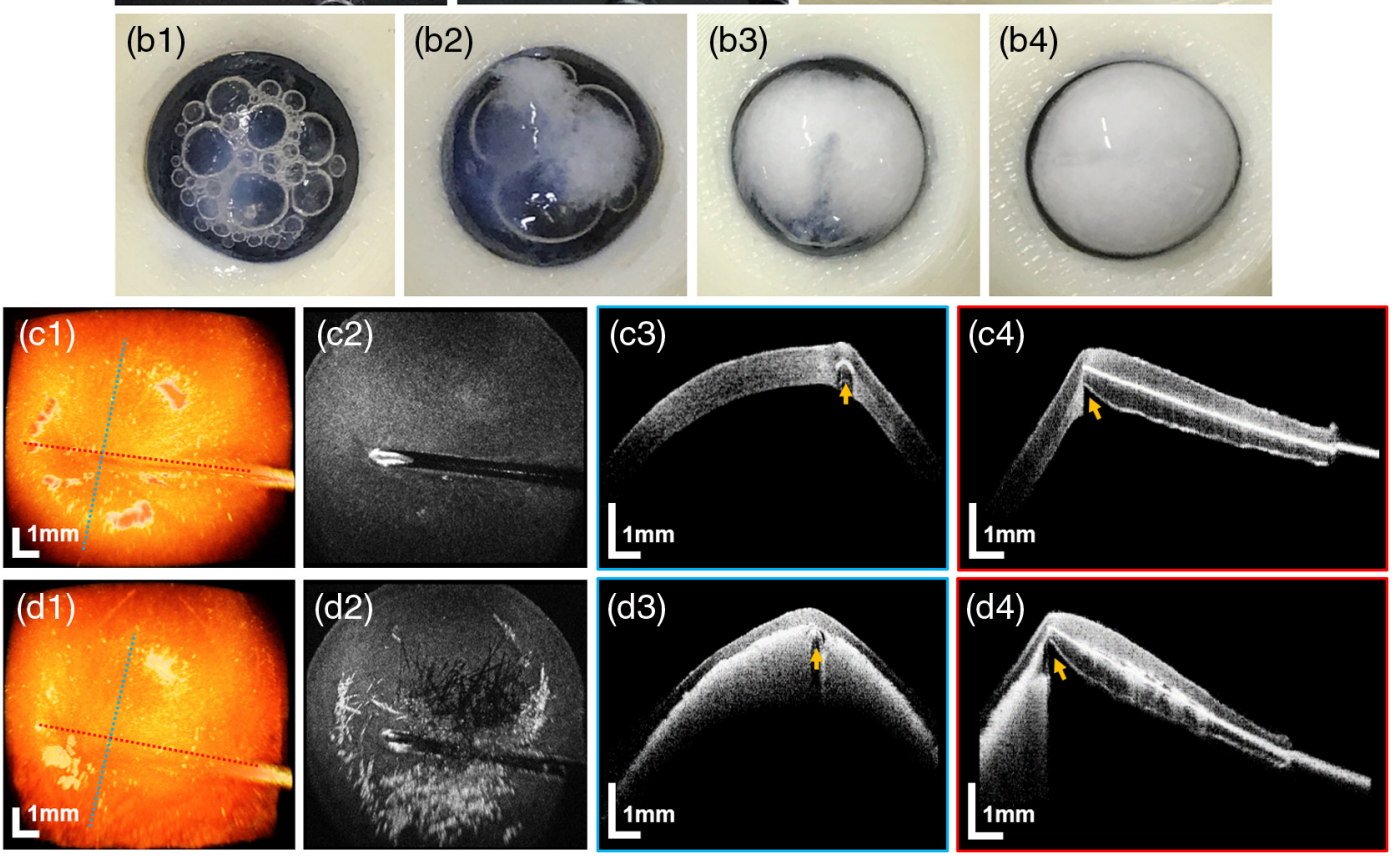

Fig. 4 Feasibility study of bubble formation with position-guided needle through 3-D OCT images on rabbit eyes. (a1)-(a3) Process of inserting a needle before air injection into the rabbit eye. (b1), (b2) Failed big-bubble cases after air injection due to over- and under-penetration of the needle, respectively. (b3), (b4) Successful big-bubble cases after air injection $\sim 100-\mu \mathrm{m}$ position. (c1)-(c4) 3-D OCT image, en-face image, and cross-sectional images in different views before air injection, (d1)(d4) after air injection.

the cornea in both success and failure cases of big-bubble formation. The blue and red lines in Figs. 4(c) and 4(d) show the cross-sectional image perpendicular and parallel to the needle, respectively. After the big-bubble technique was applied, it was found that more than half of the stroma layer was detached and air entered underneath, as shown in Fig. 4(d). From Fig. 4, we realized that DALK was successfully operated without any tissue damage by means of the position-guided needle.

\section{Discussion and Conclusion}

In this study, we introduced the M-mode OCT needle, which enables us to improve the surgical yield of DALK while minimizing fatal perforations due to unexpected needle movement. The STC fiber, operating as an optical sensor in the surgical needle, successfully traced the position of the needle end in the real time. This was confirmed by the phantom study. Through the animal model, we identified that the position-guided surgical needle offered the accurate feedback of needle placement inside the cornea, which enabled stable air bubble formation.
Furthermore, displaying the residual thickness from the desired position as an M-mode OCT image could deliver comprehensive information to the user throughout the entire surgery. Thus, experimental results suggest that our device and corresponding new surgical protocol have great potential for use in DALK as well as other microsurgeries.

However, there are several issues that need to be considered for further usage. No structural change was made, in this study, to the standard 26-G syringe needle's body in keeping its original robustness for reliably penetrating soft tissue like corneal layers. However, there are possibilities of broken tip of the STC fiber due to the pressure during the insertion process into the harder tissues. Fortunately, these could be improved by blocking the end of needle partially to reduce the external forces while maintaining the passage for airflow. In addition, we have found smearing artifact due to nonuniform movement or abrupt stops encountered during data acquisition. This could possibly confuse users for finding the location of the needle. However, there are few methods reported in public to correct these artifacts in HW/SW wise. ${ }^{33-35}$ 
The current device can be enhanced and matured by adapting other advanced engineering techniques. Our surgical needle can readily be integrated into an existing intraoperative OCT with a surgical microscope, or implemented as a handheld surgical tool. Previous studies have reported successful adaptations of OCT as a surgical tool and its potential for monitoring the surgical procedures. ${ }^{15,20,28}$ Since OCT is a modular system, the imaging path of the OCT system can either be shared for intraoperative OCT imaging or a sensor. Few studies have described the feasibility of intraoperative OCT to DALK. ${ }^{18,19,23}$ Thus, the combination of existing intraoperative OCT and our device would make a great impact on microsurgery, which requires the precise manipulation of a needle as well as the continuous observation of a surgical region.

Our device platform offers real-time depth tracking sensor. We believe this can be transformed into a tremor compensating device $^{36}$ by utilizing the motion feedback actuator and software based on our position information. In addition, current position information can be converted into a sound or alarm to make it a more user friendly surgical device. Thus, a fully developed and ergonomically engineered surgical needle would find numerous applications. The precise depth information of a needle with morphologic data could be required in microsurgery as well as in drug delivery. ${ }^{37}$

\section{Disclosures}

The authors declare that there are no conflicts of interest related to this article.

\section{Acknowledgments}

This work was supported by the Technology Innovation Program (No. 10047943) through the Ministry of Trade, Industry and Energy of Korea, the Basic Science Research Program (NRF-2014R1A1A1006183) through the National Research Foundation of Korea, and the 2017 Research Fund (No. 1.170009.01) of Ulsan National Institute of Science and Technology (UNIST). The authors would like to thank Jin U. Kang at Johns Hopkins University for his advice and comments related to this work.

\section{References}

1. J. M. Richard, D. Paton, and A. R. Gasset, "A comparison of penetrating keratoplasty and lamellar keratoplasty in the surgical management of keratoconus," Am. J. Ophthalmol. 86(6), 807-811 (1978).

2. G. R. Melles et al., "A quick surgical technique for deep, anterior lamellar keratoplasty using visco-dissection," Cornea 19(4), 427-432 (2000).

3. M. Kawashima et al., "Comparison of deep lamellar keratoplasty and penetrating keratoplasty for lattice and macular corneal dystrophies," Am. J. Ophthalmol. 142(2), 304-309 (2006).

4. I. Bahar et al., "Comparison of three different techniques of corneal transplantation for keratoconus," Am. J. Ophthalmol. 146(6), 905-912 (2008).

5. A. Anshu et al., "Outcomes of therapeutic deep lamellar keratoplasty and penetrating keratoplasty for advanced infectious keratitis: a comparative study," Ophthalmology 116(4), 615-623 (2009).

6. W. J. Reinhart et al., "Deep anterior lamellar keratoplasty as an alternative to penetrating keratoplasty a report by the American Academy of Ophthalmology," Ophthalmology 118(1), 209-218 (2011).

7. M. Anwar and K. D. Teichmann, "Deep lamellar keratoplasty: surgical techniques for anterior lamellar keratoplasty with and without baring of Descemet's membrane," Cornea 21(4), 374-383 (2002).

8. M. Anwar and K. D. Teichmann, "Big-bubble technique to bare Descemet's membrane in anterior lamellar keratoplasty," $J$. Cataract Refractive Surg. 28(3), 398-403 (2002).
9. S. L. Watson et al., "Comparison of deep lamellar keratoplasty and penetrating keratoplasty in patients with keratoconus," Ophthalmology 111(9), 1676-1682 (2004).

10. L. Fontana, G. Parente, and G. Tassinari, "Clinical outcomes after deep anterior lamellar keratoplasty using the big-bubble technique in patients with keratoconus," Am. J. Ophthalmol. 143(1), 117-124 (2007).

11. A. Leccisotti, "Descemet's membrane perforation during deep anterior lamellar keratoplasty: prognosis," J. Cataract Refractive Surg. 33(5), $825-829$ (2007).

12. H. M. Anwar, A. El-Danasoury, and A. N. Hashem, "The use of fibrin glue to seal Descemet membrane microperforations occurring during deep anterior lamellar keratoplasty," Cornea 31(1), 1193-1196 (2012).

13. K. A. Knutsson, P. Rama, and G. Paganoni, "Modified big-bubble technique compared to manual dissection deep anterior lamellar keratoplasty in the treatment of keratoconus," Acta Ophthalmol. 93(5), 431-438 (2015).

14. M. A. Javadi et al., "Visual outcomes of successful versus failed big-bubble deep anterior lamellar keratoplasty for keratoconus," J. Ophthalmic Vision Res. 11(1), 32-36 (2016).

15. G. Geerling et al., "Intraoperative 2-dimensional optical coherence tomography as a new tool for anterior segment surgery," Arch. Ophthalmol. 123(2), 253-257 (2005).

16. R. B. Kucumen et al., "Intraoperative use of AS-OCT during intrastromal corneal ring segment implantation," Ophthalmic Surg. Lasers Imaging 43(6), S109-S116 (2012).

17. V. Scorcia et al., "Anterior segment optical coherence tomographyguided big-bubble technique," Ophthalmology 120(3), 471-476 (2013).

18. L. De Benito-Llopis et al., "Intraoperative anterior segment optical coherence tomography: a novel assessment tool during deep anterior lamellar keratoplasty," Am. J. Ophthalmol. 157(2), 334-341 (2014).

19. P. Steven et al., "Optimising deep anterior lamellar keratoplasty (DALK) using intraoperative online optical coherence tomography (iOCT)," Br. J. Ophthalmol. 98(7), 900-904 (2014).

20. J. P. Ehlers et al., "Integration of a spectral domain optical coherence tomography system into a surgical microscope for intraoperative imaging," Invest. Ophthalmol. Visual Sci. 52(6), 3153-3159 (2011).

21. Y. K. Tao, S. K. Srivastava, and J. P. Ehlers, "Microscope-integrated intraoperative OCT with electrically tunable focus and heads-up display for imaging of ophthalmic surgical maneuvers," Biomed. Opt. Express 5(6), 1877-1885 (2014).

22. J. P. Ehlers, P. K. Kaiser, and S. K. Srivastava, "Intraoperative optical coherence tomography using the RESCAN 700: preliminary results from the DISCOVER study," Br. J. Ophthalmol. 98(10), 1329-1332 (2014).

23. N. D. Pasricha et al., "Needle depth and big-bubble success in deep anterior lamellar keratoplasty: an ex vivo microscope-integrated OCT study," Cornea 35(11), 1471-1477 (2016).

24. J. P. Ehlers et al., "Integrative advances for OCT-guided ophthalmic surgery and intraoperative OCT: microscope integration, surgical instrumentation, and heads-up display surgeon feedback," PLoS One 9(8), e105224 (2014).

25. J. P. Ehlers et al., "Visualization of real-time intraoperative maneuvers with a microscope-mounted spectral domain optical coherence tomography system," Retina 33(1), 232-236 (2013).

26. O. M. Carrasco-Zevallos et al., "Live volumetric (4D) visualization and guidance of in vivo human ophthalmic surgery with intraoperative optical coherence tomography," Sci. Rep. 6(1), 31689 (2016).

27. X. Li et al., "Imaging needle for optical coherence tomography," Opt. Lett. 25(20), 1520-1522 (2000).

28. S. Han et al., "Handheld forward-imaging needle endoscope for ophthalmic optical coherence tomography inspection," J. Biomed. Opt. 13(2), 020505 (2008).

29. B. C. Quirk et al., "In situ imaging of lung alveoli with an optical coherence tomography needle probe," J. Biomed. Opt. 16(3), 036009 (2011).

30. D. Lorenser et al., "Ultrathin side-viewing needle probe for optical coherence tomography," Opt. Lett. 36(19), 3894-3896 (2011).

31. L. Scolaro et al., "High-sensitivity anastigmatic imaging needle for optical coherence tomography," Opt. Lett. 37(24), 5247-5249 (2012).

32. J. Lee et al., "Ultra-thin and flexible endoscopy probe for optical coherence tomography based on stepwise transitional core fiber," Biomed. Opt. Express. 6(5), 1782-1796 (2015). 
33. P. Pande et al., "Sensor-based technique for manually scanned hand-held optical coherence tomography imaging," J. Sens. 2016, 8154809 (2016).

34. X. Liu, Y. Huang, and J. U. Kang, "Distortion-free freehand-scanning OCT implemented with real-time scanning speed variance correction," Opt. Express 20(15), 16567-16583 (2012).

35. A. Ahmad et al., "Cross-correlation-based image acquisition technique for manually-scanned optical coherence tomography," Opt. Express 17(10), 8125-8136 (2009).

36. C. Song, P. L. Gehlbach, and J. U. Kang, "Active tremor cancellation by a 'smart' handheld vitreoretinal microsurgical tool using swept source optical coherence tomography," Opt. Express. 20(21), 23414-23421 (2012).

37. M. R. Prausnitz and R. Langer, "Transdermal drug delivery," Nat. Biotechnol. 26(11), 1261-1268 (2008).

Sungwon Shin received his MSc degree in control and measurement engineering from University of Ulsan in 2008. Currently, he is pursuing his $\mathrm{PhD}$ in the Department of Biomedical Engineering at Ulsan National Institute of Science and Technology. Since the spring of 2014, he has been with Dr. Woonggyu Jung's Transitional Biophotonics Laboratory. His research interests include utilizing biomedical imaging for the detection of diseases and developing new surgical guidance tools for clinical applications.

Jung Kweon Bae received his BSc degree in electrical engineering from the University of Illinois at Urbana Champaign in 2013. Currently, he is pursuing his PhD in the Department of Biomedical Engineering at Ulsan National Institute of Science and Technology. Since the fall of 2013, he has been with Dr. Woonggyu Jung's Transitional Biophotonics Laboratory. His research interests include biomedical imaging device, mobile-based imaging, point-of-care diagnostics, mobile health, and clinical applications.

Sucbei Moon received his MS and PhD degrees from Gwangju Institute of Science and Technology in 2002 and 2007, respectively. Since he joined the faculty of physics, Kookmin University in Seoul, South Korea, in 2011, he has been exploring various topics of applied optics, with particular emphasis on optical imaging technologies. His current research interest includes the optical coherence tomography, catheter-based imaging, and fiber-optic imaging techniques.

Woonggyu Jung is an associate professor of biomedical engineering at Ulsan National Institute of Science and Technology. He received his $\mathrm{PhD}$ in biomedical engineering from the University of California, Irvine, in 2008. He has published various papers in the field of biomedical optics and biophotonics. His research interests include medical imaging systems, point-of-care diagnostics, label-free imaging, optical stimulation, and clinical applications.

Biographies for the other authors are not available. 